\section{Nuclear Astrophysics}

held to be strong evidence in its favour, especially in view of the predicted deuterium abundance. The results are so important that we devote some time in our symposium to looking deeper into the simple picture and ask what it is that determines, in the very early stages of the Universe, the values of the baryon number, the lepton numbers and the temperature, and to what extent the simple picture may be altered, yet still retain the essential features of the nucleosynthesis era.

Paradoxically, because there is only one Universe and despite the central position of the Hot Big Bang picture, we also look seriously at alternative models of the Universe which seek to explain the origin of the light elements etc. in other ways. For example there is some current interest in cold or tepid universes in which neutrino degeneracy, black holes and super-massive stars all play a role. There are also indications today that there is a lot of matter in the Universe that is only visible in the $\mathrm{X}$-ray region. This might upset the simple Hot Big Bang picture and it could be that the Universe is playing cat and mouse with us in our attempts to explain it - but that only makes the game more exciting.

\section{A. Edwards}

\section{Heavy Ion Physics}

important contributions to the investigation of transuranium elements have come as well as fundamental results in nuclear and atomic reaction studies.

At present much activity is under way at the new heavy ion accelerator UNILAC of the GSI near Darmstadt which has been in operation since early 1976. New facilities in Europe will be in operation in the near future, such as the GANIL project near Caen (France), the VICKSI project at Berlin, and the $30 \mathrm{MV}$ tandem Van de Graaff accelerator at the Daresbury Laboratory in England. A review of the present state of these facilities and their comparison will be given at the Conference.

The production of elements beyond

uranium is one of the most interesting aims in the whole field because its study should reveal the criteria of nuclear stability and the construction principles of nuclei. Unfortunately, progress so far has been slow and the nuclear physics community has been trying hard to find new ways to reach this goal. The nuclear reaction mechanisms ruling the interactions of complex nuclei have to be studied and proved at already known nuclei which are presently very thoroughly investigated to find a feasible way for the production of superheavy elements. In particular, the behaviour of nuclei with high angular momenta and the Coulomb interactions are being studied extensively.

Heavy ion atomic physics is very closely related to the fundamental test of quantum electro-dynamics of very strong fields. Those fields can be produced in the interpenetration of heavy-particle atomic-shell systems giving rise to the formation of an atomic shell system of the combined atom $Z_{1}+Z_{2}$. During the collision,

\title{
Symposium E 2: Quarks
}

The notion of "Quarks" dominates today the field of High Energy and Particle Physics. Under the impulse of the very simple idea (Gell-Mann and Zweig, 1964) that hadrons are made out of much smaller constituents, high energy physics has explored vast new domains which have disclosed very fascinating structures.

This Symposium intends to focus on those areas of High Energy physics where the quark idea has found its most fruitful applications and has led to in-depth experimentation. Today we know a vast number of particles with different charges, spins and parities and all can be classified according to the idea that they are constituted from a quark-antiquark pair (mesons) and three quarks (baryons). The recent discovery of the $\mathrm{J} / \mathrm{psi}$ has only strengthened our belief in the relevance of quarks, for it has become clear that their spectrum too could be understood according to the simple quark model.

Another very important area, where quarks play a central role is the field of high energy neutrino and electron scattering on a nuclear target. The results of many different experiments can be explained by thinking of hadronic matter as made out of a number of pointlike constituents : the par- vacancies in the strong bound $\mathrm{K}$ shells can be produced which dive into the negative energy continuum. There the spontaneous production of positrons has been observed. Furthermore, X-ray transitions of these socalled quasi-molecules have already been observed which belong to the united atom. During these interactions also, atomic systems of superheavy atoms can be formed. The transitions in such a system reveal features of superheavy elements and their atomic properties. The well-known Coulomb interaction allows also the development of theoretical models and thus predictions which could be tested experimentally. However, a great number of detailed problems also have to be solved before a consistent picture can be drawn. A number of reviews will be devoted to these problems.

The implantation of heavy particles into solid material opens new aspects of applied physics and technology.

K. Bethge

tons (Feynman 1969), which scatter electromagnetic and weak probes in a way similar to the well known leptons (electrons and muons). What is amazing is that partons and quarks seem to show a great deal of properties.

This fact, however, brings us to a very critical situation. If matter is made out of such simple objects, we should be able to see them under appropriate experimental conditions (i.e. in deep inelastic or high $\mathrm{p}_{\mathrm{T}}$ scattering). But so far the hadronic constituents have eluded the most ingenious and tenacious searches. It is this paradoxical situation that has induced many theorists to think that quarks are basically "confined", and has encouraged a great deal of theoretical effort on the quark-"confinement" problem, which is the really central problem of modern high energy theoretical physics.

The Symposium on Quarks will deal with all these problems, in order to make the point about the status of our understanding of this puzzling and fascinating idea. 